\title{
Hilfe zur Teilhabe
}

\section{In der Begegnung von Menschen bilden sich die Werte einer Gesellschaft}

\author{
Donata Freifrau Schenck zu Schweinsberg
}

Der Begriff der "Werte " gewinnt, seit er Ende der 1990er Jahre von Denkern wie Hans Jonas oder Hartmut von Hentig neu beleuchtet wurde, in der gesamtgesellschaftlichen Diskussion an Bedeutung. Begründet scheint dies in dem sich ausbreitenden Gefühl vieler Menschen, dass Phänomene, wie soziale Rücksichtslosigkeit oder verunsichernde Orientierungsschwierigkeiten bei wichtigen Lebensentscheidungen darin begründet liegen, dass Menschen nicht mehr ausreichend an universelle Werte gebunden sind.

Die maßgeblichen Werte einer Gesellschaft, die allgemein akzeptiert sind und gelten, drücken sich aus in Normen, also Vorschriften und Gesetzen - geschriebenen und ungeschriebenen. Das Grundgesetz, das Strafgesetzbuch und das Bürgerliche Gesetzbuch sind die prominentesten Verschriftlichungen zur Geltendmachung von Werten in Deutschland. Darin wird all das geregelt, was ein Gesetz »wert « ist.

\section{Wertbindung als Auftrag}

Doch es reicht offenbar nicht aus, dass Gesetze gelten. Werte, die über das gesetzlich Geregelte hinausgehen, müssen gelebt werden. Dies gelingt nur, wenn Menschen sich Werten verpflichtet und an sie gebunden fühlen. Dies wiederum setzt einen Wertebindungsprozess voraus. Die Vorstellung eines Bindungsprozesses macht das Thema "Werte « auch zu einer Aufgabe der Pädagogik und Sozialarbeit und damit zu einem Thema der Freien Wohlfahrtspflege und des Deutschen Roten Kreuzes (DRK).

Der bekannteste Wert, mit dem das Rote Kreuz verbunden wird, ist sein erster Grundsatz »Menschlichkeit «. Im Sinne von Nächstenliebe $(\mathbb{S} 1$ Absatz 3 der Satzung des DRK) ist Mitmenschlichkeit der herausragende Wert des Roten Kreuzes. Menschen helfen Menschen - bedingungslos. Dies ist der Kern des Roten
Kreuzes. Die Gestaltung eines menschlichen Miteinanders ist Auftrag und Aufgabe des Roten Kreuzes. Im Roten Kreuz begegnen sich hilfebedürftige und helfende Menschen. Beide gemeinsam erleben und erzeugen Menschlichkeit. Das Rote Kreuz ist wertegebunden (Grundsätze, Satzung, Leitbild) und wertevermittelnd (Verbreitungsarbeit, Satzung des DRK \2 Absatz 2). Es ist Verbreiter und Anwender seiner Grundsätze und des humanitären Völkerrechts. Durch sie ist das Deutsche Rote Kreuz immer an etwas über sich selbst hinaus Weisendes gebunden und zunächst ausschließlich dem Wohl des einzelnen Menschen verpflichtet.

\section{Werte erleben}

Universelle Werte, also ethische Prinzipien wie die Menschenrechte, werden in fast allen Nationen und Kulturen der Welt anerkannt, aber unterschiedlich gelebt. Die für die Umsetzung eines Wertes notwendigen Interpretationen der universellen Werte werden in der postmodernen und pluralistischen Gesellschaft der Bundesrepublik in hohem Maße von den sich schnell vollziehenden gesellschaftlichen Veränderungen mitbestimmt.

Nicht nur Wertverständnisse und Normen haben sich verändert, sondern auch das Verständnis ihrer Geltung. Während in früheren Generationen die unumstößliche Regelbefolgung leitend war, prüft die junge Generation, ob die Befolgung der Regel in der konkreten Situation ihren Sinn erfüllt und dabei nicht in Konflikt mit anderen Werten und Normen gerät. Die multikulturelle, heterogene Gesellschaft der Bundesrepublik bringt verschiedene Moralverständnisse derer mit sich, die in Familie, Nachbarschaft und Stadtteil Gemeinsames erleben und miteinander handeln (müssen). Vor diesem Hintergrund ist jeder Einzelne hinsichtlich seiner Orientierungsfähigkeit und Ei- 
genständigkeit herausgefordert. Die in früherer Zeit starke Außenleitung durch strikte Regeln und Normen wurde abgelöst von einer stärkeren Innenleitung. Jene Innenleitung bedarf einer durch Einsicht und Überzeugung entstandenen Selbstbindung des Einzelnen an die gemeinsam geteilten Werte der Gemeinschaft, in der er lebt.

\section{Wie Selbstbindung gelingen kann}

Wie bei jedem Bindungs- und Bildungsprozess nimmt auch dieser Selbstbindungsprozess seinen Anfang in der frühen Kindheit. In dieser Zeit werden voraussetzende Kompetenzen erworben, wie Strategien, sich in einem ersten Schritt an Menschen und in einem zweiten an Ideen, Werte, Ideale zu binden, oder die Konfliktlösungsstrategien, die notwendig sind, um die inter- und intrapersonellen Wertekonflikte zu bewältigen. Moralisches Wissen entwickeln Kinder im Alter von vier bis fünf Jahren. Neben den voraussetzenden Kompetenzen ist die moralische Motivation ein weiterer Faktor für die Selbstbindung an Werte. Die moralische Motivation nimmt ihren Anfang in der mittleren Kindheit und bleibt Entwicklungsaufgabe im Jugendalter.

Was ist es konkret, das Menschen motiviert, sich an Werte zu binden? Neben der erlebten Achtung der Person, insbesondere durch die Bezugspersonen, ist die Entwicklung eines Gefühls der Zugehörigkeit zur Familie, zur Kinder- und Jugendgruppe, zur Nachbarschaft und zur Gesellschaft ein wichtiger Motivationsfaktor. Von unschätzbarer Bedeutung ist dabei die Erfahrung der Teilhabe an den Prozessen in diesen Gruppen.

\section{Wirkung der Wohlfahrtsarbeit - Helfer und Hilfenehmer im direkten Kontakt}

Menschen, die in schwierigen Lebenssituationen Hilfe von anderen Menschen annehmen müssen, tun das in der Regel nicht gern. Zugeben zu müssen, dass man bestimmte Dinge seines Lebens nicht mehr allein bewältigen kann, berührt das Selbstwertgefühl und Selbstbewusstsein und kann dazu führen, dass man sich als in der Schuld des Helfenden wahrnimmt. Eine für den Hilfe Empfangenden in die- sem Sinne besondere Situation stellt die Hilfe durch ehrenamtlich Helfende eines Wohlfahrtsverbands dar.

Die ehrenamtlich Helfenden gehen in ihrer Hilfeleistung keiner Erwerbsarbeit nach, sondern folgen ihrer persönlichen ideellen Motivation, wie beispielsweise einen gesellschaftlichen Beitrag zu leisten, etwas Gutes zu tun, Menschlichkeit umzusetzen. Die ehrenamtlich Helfenden tun damit auch etwas für ihr Selbstverständnis als Person. Sie setzen ihre Ideale und Werte um, indem sie Hilfe leisten. Die ehrenamtlich Helfenden helfen nicht innerhalb ihrer Familien oder ihrer Freundeskreise, sondern Menschen, zu denen sie zunächst in keiner Beziehung stehen und denen gegenüber damit auch keine persönliche Verpflichtung zur Hilfe besteht. Die Helfenden helfen freiwillig aus innerer Motivation heraus.

Der Mensch, dem geholfen wird, nimmt wahr, dass die Helfenden sich ihm freiwillig zuwenden und sich die Hilfe nicht an ihn als bekannte Person richtet, sondern einfach an ihn als Menschen. Der Hilfe Empfangende erlebt, dass der ihm Helfende seine Wertvorstellungen durch Hilfe umsetzt. Es entsteht somit eine Situation des Gewinns für beide Seiten, für den Helfenden genauso wie für den Hilfe Empfangenden. Hier fühlt sich keiner in des Anderen Schuld, ein Gefühl der Gleichwertigkeit entsteht, insbesondere beim Hilfe Empfangenden. Der Hilfe Empfangende und der Helfende werden durch die spezifischen Bedingungen ihres Zusammentreffens in ihren Werte bildenden Prozessen gestärkt.

Ein Hilfe empfangender Mensch, der "Menschlichkeit « am eigenen Leib als positiven Wert erfahren hat und erleben konnte, wie die Umsetzung dieses Wertes dem ehrenamtlich Helfenden gewissermaßen Erfüllung bringt, wird dadurch motiviert, auch sich selbst an diesen Wert zu binden. Als Raum, in dem Mitmenschlichkeit gelebt und erlebt wird, leistet das DRK somit seinen spezifischen Beitrag zur Wertebildung in der Gesellschaft und zur Wertebindung des Einzelnen.

\section{Gutes Geld für gute Zwecke}

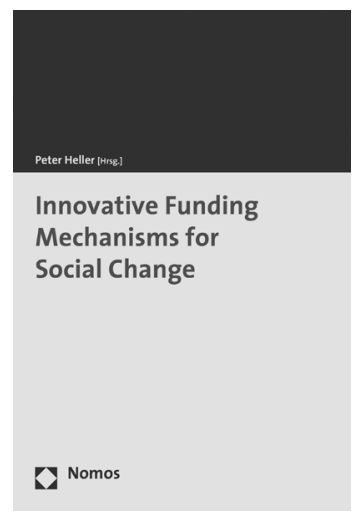

Innovative Funding Mecha-
nisms for Social Change

Herausgegeben von

Dr. Peter W. Heller

2009, 144 S., brosch., 24,- $€$,

ISBN 978-3-8329-3948-9

Wo die soziale Sicherung des Staates versagt, findet die Zivilgesellschaft ihre eigenen Lösungen. In vielen Ländern haben zivilgesellschaftliche Organisationen mehr Arbeitsplätze geschaffen als der öffentliche und der private Sektor. Um den damit verbundenen Herausforderungen gerecht zu werden, orientieren sich soziale Pioniere an den erfolgreichen Geschäftsmodellen und Management-Strategien der privaten Unternehmen, sie werden zu „social entrepreneurs". Aber oft fehlen innen die finanziellen Mittel, um ihr soziales Unternehmen auf einen stabilen Wachstumskurs zu bringen.

Dieses Buch untersucht das Potential maßgeschneiderter sozialer Investmentfonds und Investitionsstrategien von Stiftungen, um auf neuen Wegen das fehlende Kapital bereitzustellen.

\section{Nomos}

Bitte bestellen Sie im Buchhandel oder versandkostenfrei unter $>$ www.nomos-shop.de 